\title{
Existence and approximation for a solution of a generalized equilibrium problem on the dual space of a Banach space
}

Pongrus Phuangphoo ${ }^{1,2}$ and Poom Kumam ${ }^{\text {* }}$

Dedicated to Prof. W Takahashi on the occasion of his 70th birthday

"Correspondence:

poom.kum@kmutt.ac.th

'Department of Mathematics,

Faculty of Science, King Mongkut's University of Technology Thonburi (KMUTT), Bang Mod, Thung Khru, Bangkok, 10140, Thailand

Full list of author information is

available at the end of the article

\begin{abstract}
In this paper, we first prove the existence of a solution for a generalized equilibrium problem with a bifunction defined on the dual space in a Banach space setting. Second, by the virtue of this result, we construct the hybrid projection method for solving a solution of a generalized equilibrium problem. Consequently, we establish the strong convergence theorem by using sunny generalized nonexpansive retraction in the dual of Banach spaces.

MSC: 47H09; 47H10; 47J25
\end{abstract}

Keywords: a generalized equilibrium problem; a bifunction defined on the dual space; the hybrid projection method

\section{Introduction}

Let $\mathbb{R}$ be the set of real numbers. Let $E$ be a real Banach space with the norm $\|\cdot\|$, and $\langle\cdot, \cdot\rangle$ is the dual pair between $E$ and $E^{*}$, where $E^{*}$ is the dual space of $E$. Let $C$ be a closed and convex subset of a real Banach space $E$ with the dual space $E^{*}$, and let $C^{*}$ be a closed and convex subset of $E^{*}$. We recall the following definitions:

(1) A mapping $A: C \rightarrow E^{*}$ is said to be monotone if for each $x, y \in C$ such that

$$
\langle x-y, A x-A y\rangle \geq 0 .
$$

(2) A mapping $A: C \rightarrow E^{*}$ is said to be $\delta$-strongly monotone if there exists a constant $\delta>0$ such that

$$
\langle x-y, A x-A y\rangle \geq \delta\|x-y\|^{2}, \quad \forall x, y \in C .
$$

(3) A mapping $A: C \rightarrow E^{*}$ is said to be $\delta$-inverse strongly monotone if there exists a constant $\delta>0$ such that

$$
\langle x-y, A x-A y\rangle \geq \delta\|A x-A y\|^{2}, \quad \forall x, y \in C .
$$

(4) A mapping $A: C^{*} \rightarrow E$ is said to be skew monotone if for each $x^{*}, y^{*} \in C^{*}$ such that

$$
\left\langle A x^{*}-A y^{*}, x^{*}-y^{*}\right\rangle \geq 0 .
$$


(5) A mapping $A: D(A) \subset E^{*} \rightarrow E$ is said to be $\alpha$-inverse strongly skew monotone if there exists a constant $\alpha>0$ such that

$$
\left\langle A x^{*}-A y^{*}, x^{*}-y^{*}\right\rangle \geq \alpha\left\|A x^{*}-A y^{*}\right\|^{2}, \quad \forall x^{*}, y^{*} \in D(A) .
$$

(6) A mapping $A: D(A) \subset E^{*} \rightarrow E$ is said to be hemicontinuous if for all $x^{*}, y^{*} \in D(A)$, the mapping $f$ of $[0,1]$ into $E$ defined by $f(t)=A\left(t x^{*}+(1-t) y^{*}\right)$ is continuous.

Let $C$ be a nonempty, closed and convex subset of $E$, and let $J$ be the duality mapping from $E$ into $E^{*}$ such that $J(C)$ is closed and convex of $E^{*}$, let us assume that a bifunction $F: J(C) \times J(C) \rightarrow \mathbb{R}$ satisfies suitable conditions, and $A: C^{*} \rightarrow E$ is a skew monotone operator from $J(C)$ into $E$.

The generalized equilibrium problem is to find $\hat{z} \in C$ such that

$$
F(J \hat{z}, J y)+\langle A J \hat{z}, J y-J \hat{z}\rangle \geq 0, \quad \forall y \in C
$$

The set of solutions of $(1.1)$ is denoted by $\operatorname{GEP}(F, A)$, that is,

$$
\operatorname{GEP}(F, A)=\{\hat{z} \in C: F(J \hat{z}, J y)+\langle A J \hat{z}, J y-J \hat{z}\rangle \geq 0, \forall y \in C\}
$$

If $A \equiv 0$, then problem (1.1) reduces to the equilibrium problem, which is to find $\hat{z} \in C$ such that

$$
F(J \hat{z}, J y) \geq 0, \quad \forall y \in C
$$

The set of solutions of problem (1.3) is denoted by $\operatorname{EP}(F)$, that is,

$$
\operatorname{EP}(F)=\{\hat{z} \in C: F(J \hat{z}, J y) \geq 0, \forall y \in C\}
$$

The above formulation (1.3) was considered in Takahashi and Zembayashi [1], and they proved a strong convergence theorem for finding a solution of the equilibrium problem (1.3) in Banach spaces.

If $F \equiv 0$, then problem (1.1) reduces to variational inequality, which is to find $\hat{z} \in C$ such that

$$
\langle A J \hat{z}, J y-J \hat{z}\rangle \geq 0, \quad \forall y \in C .
$$

The set of solutions of problem (1.5) is denoted by $\operatorname{VI}(J(C), A)$, that is,

$$
\mathrm{VI}(J(C), A)=\{\hat{z} \in C:\langle A J \hat{z}, J y-J \hat{z}\rangle \geq 0, \forall y \in C\}
$$

In the sequel, let $T: C \rightarrow C$ be a mapping, we denote by Fix $(T)$ the set of fixed points of $T$, that is,

$$
\operatorname{Fix}(T)=\{x \in C: T x=x\}
$$

We denote the strong convergence, weak convergence $\left\{x_{n}\right\}$ by $x_{n} \rightarrow x, x_{n} \rightarrow x$, respectively. 
Let $C$ be a nonempty, closed subset of a smooth, strictly convex and reflexive Banach space $E$ such that $J(C)$ is closed and convex. For solving the equilibrium problem, let us assume that a bifunction $F: J(C) \times J(C) \rightarrow \mathbb{R}$ satisfies the following conditions:

(DA1) $F\left(x^{*}, x^{*}\right)=0$ for all $x^{*} \in J(C)$;

(DA2) $F$ is monotone, i.e., $F\left(x^{*}, y^{*}\right)+F\left(y^{*}, x^{*}\right) \leq 0$ for all $x^{*}, y^{*} \in J(C)$;

(DA3) for all $x^{*}, y^{*}, z^{*} \in J(C)$,

$$
\limsup _{t \downarrow} F\left(t z^{*}+(1-t) x^{*}, y^{*}\right) \leq F\left(x^{*}, y^{*}\right)
$$

(DA4) for all $x^{*} \in J(C), F\left(x^{*}, \cdot\right)$ is convex and lower semicontinuous.

The following result is in Blum and Oettli [2], and see the proof in [3].

Definition 1.1 Let $E$ be a Banach space. Then,

(1) $E$ is said to be strictly convex if $\frac{\|x+y\|}{2}<1$ for all $x, y \in U_{E}=\{z \in E:\|z\|=1\}$ with $x \neq y$.

(2) $E$ is said to be uniformly convex if for each $\epsilon \in(0,2]$, there exists $\delta>0$ such that $\frac{\|x+y\|}{2} \leq 1-\delta$ for all $x, y \in U_{E}$ with $\|x-y\|>\epsilon$.

(3) $E$ is said to be smooth if the limit (1.8)

$$
\lim _{t \rightarrow 0} \frac{\|x+t y\|-\|x\|}{t}
$$

exists for each $x, y \in U_{E}$.

(4) $E$ is said to be uniformly smooth if the limit (1.8) is attained uniformly for all $x, y \in U_{E}$.

(5) $E$ is said to have uniformly Gâteaux differentiable norm if for all $y \in U(E)$, the limit (1.8) converges uniformly for $x \in U_{E}$.

Definition 1.2 Let $E$ be a Banach space. Then a function $\rho_{E}: \mathbb{R}^{+} \rightarrow \mathbb{R}^{+}$is said to be the modulus of smoothness of $E$ if

$$
\rho_{E}(t)=\sup \left\{\frac{\|x+y\|+\|x-y\|}{2}-1:\|x\|=1,\|y\|=t\right\} .
$$

(1) $E$ is said to be smooth if $\rho_{E}(t)>0, \forall t>0$.

(2) $E$ is said to be uniformly smooth if and only if $\lim _{t \rightarrow 0^{+}} \frac{\rho_{E}(t)}{t}=0$.

Definition 1.3 Let $E$ be a Banach space. Then the modulus of convexity of $E$ is the function $\delta_{E}:[0,2] \rightarrow[0,1]$ defined by

$$
\delta_{E}(\epsilon)=\inf \left\{1-\left\|\frac{x+y}{2}\right\|:\|x\| \leq 1,\|y\| \leq 1 ;\|x-y\| \geq \epsilon\right\} .
$$

(1) $E$ is said to be uniformly convex if and only if $\delta_{E}(\epsilon)>0$ for all $\epsilon \in(0,2]$.

(2) Let $p$ be a fixed real number $p>1$. Then $E$ is said to be $p$-uniformly convex if there exists a constant $c>0$ such that $\delta_{E}(\epsilon) \geq c \epsilon^{p}$ for all $\epsilon \in[0,2]$. 
Observe that every $p$-uniformly convex is uniformly convex. One should note that no Banach space is $p$-uniformly convex for $1<p<2$. It is well known that a Hilbert space is 2-uniformly convex and uniformly smooth.

For any $p>1$, the generalized duality mapping $J_{p}: E \rightarrow 2^{E^{*}}$ is defined by

$$
J_{p} x=\left\{f^{*} \in E^{*}:\left\langle x, f^{*}\right\rangle=\|x\|^{p},\left\|f^{*}\right\|=\|x\|^{p-1}\right\}, \quad \forall x \in E .
$$

In particular, $J=J_{2}$ is called the normalized duality mapping. If $E$ is a Hilbert space, then $J=I$, where $I$ is the identity mapping. That is,

$$
J_{2} x=J x=\left\{f^{*} \in E^{*}:\left\langle x, f^{*}\right\rangle=\|x\|^{2}=\left\|f^{*}\right\|^{2}\right\}, \quad \forall x \in E .
$$

Remark 1.4 The basic properties below hold, see [4-6].

(1) If $E$ is a uniformly smooth real Banach space, then $J$ is uniformly continuous on each bounded subset of $E$.

(2) If $E$ is a uniformly smooth real Banach space, then $J^{*}: E^{*} \rightarrow 2^{E}$ is a normalized duality mapping on $E^{*}$, then $J^{-1}=J^{*},\left(J^{*}\right) J=I_{E}$ and $J\left(J^{*}\right)=I_{E^{*}}$, where on $I_{E}$ and $I_{E^{*}}$ are the identity mappings on $E$ and $E^{*}$, respectively.

(3) Let $E$ be a smooth, strictly convex reflexive Banach space and $J$ be the duality mapping from $E$ into $E^{*}$. Then $J^{-1}$ is also single-valued, one-to-one, onto, and it is also the duality mapping from $E^{*}$ into $E$.

(4) If $E$ is a reflexive, strictly convex Banach space, then $J^{-1}$ is hemicontinuous, that is, $J^{-1}$ is norm-to-weak ${ }^{*}$-continuous.

(5) If $E$ is a reflexive, smooth and strictly convex Banach space, then $J$ is single-valued, one-to-one, and onto.

(6) A Banach space $E$ is uniformly smooth if and only if $E^{*}$ is uniformly convex.

(7) Each uniformly convex Banach space $E$ has the Kadec-Klee property, that is, for any sequence $\left\{x_{n}\right\} \subset E$, if $x_{n} \rightarrow x \in E$, and $\left\|x_{n}\right\| \rightarrow\|x\|$, then $x_{n} \rightarrow x$.

(8) A Banach space $E$ is strictly convex if and only if $J$ is strictly monotone, that is,

$$
\left\langle x-y, x^{*}-y^{*}\right\rangle>0, \quad \text { whenever } x, y \in E, x \neq y \text {, and } x^{*} \in J x, y^{*} \in J y \text {. }
$$

(9) Both, uniformly smooth Banach space and uniformly convex Banach space, are reflexive.

(10) If $E^{*}$ is uniformly convex, then $J$ is uniformly norm-to-norm continuous on each bounded subset of $E$.

(11) If $E^{*}$ is a strictly convex Banach space, then $J$ is one-to-one, that is, $x \neq y$ implies that $J x \cap J y \neq \emptyset$.

Let $J$ be the normalized duality mapping, then $J$ is said to be weakly sequentially continuous if the strong convergence of a sequence $\left\{x_{n}\right\}$ to $x \in E$ implies the weak* convergence of a sequence $\left\{J x_{n}\right\}$ to $J x$ in $E^{*}$.

Let $E$ be a smooth and strictly convex reflexive Banach space, and let $C$ be a nonempty, closed and convex subset of $E$. We assume that the Lyapunov functional $\phi: E \times E \rightarrow \mathbb{R}^{+}$ is defined by $[6,7]$

$$
\phi(x, y)=\|x\|^{2}-2\langle x, J y\rangle+\|y\|^{2}, \quad \forall x, y \in E .
$$


Let $C$ be a nonempty, closed and convex subset of a Banach space $E$. The generalized projection [6] $\Pi_{C}: E \rightarrow C$ is defined by, for each $x \in E$,

$$
\Pi_{C}(x)=\arg \min _{y \in C} \phi(x, y) .
$$

Remark 1.5 From the definition of $\phi$. It is easy to see that

(1) $(\|x\|-\|y\|)^{2} \leq \phi(x, y) \leq(\|x\|+\|y\|)^{2}$ for all $x, y \in E$.

(2) $\phi(x, y)=\phi(x, z)+\phi(z, y)+2\langle x-z, J z-J y\rangle$ for all $x, y, z \in E$.

(3) $\phi(x, y)=\langle x, J x-J y\rangle+\langle y-x, J y\rangle \leq\|x\|\|J x-J y\|+\|y-x\|\|y\|$ for all $x, y, z \in E$.

(4) If $E$ is a real Hilbert space $H$, then $\phi(x, y)=\|x-y\|^{2}$, and $\Pi_{C}=P_{C}$ (the metric projection of $H$ onto $C$ ).

Lemma 1.6 [6, 7] If $C$ is a nonempty, closed and convex subset of a smooth and strictly convex reflexive real Banach space $E$, then

(1) for $x \in E$, and $u \in C$, one has

$$
u=\Pi_{C}(x) \quad \Leftrightarrow \quad\langle u-y, J x-J u\rangle \geq 0, \quad \forall y \in C .
$$

(2) $\phi\left(x, \Pi_{C}(y)\right)+\phi\left(\Pi_{C}(y), y\right) \leq \phi(x, y), \forall x \in C, y \in E$.

(3) $\phi(x, y)=0$ if and only if $x=y, \forall x, y \in E$.

In 2007, Takahashi and Zembayashi [1] introduced an iterative algorithm for finding a solution of an equilibrium problem with a bifunction defined on the dual space of a Banach space by using the shrinking projection method, and they established the strong convergence as follows.

Theorem TZ Let E be a uniformly convex Banach space whose norm is uniformly Gâteaux differentiable, and let $C$ be a nonempty, closed and convex subset of $E$ such that $J(C)$ is closed and convex of $E^{*}$. Assume that a mapping $F: J(C) \times J(C) \rightarrow \mathbb{R}$ satisfies the condition (DA1)-(DA4) such that $\mathrm{EP}(F) \neq \emptyset$. Let $\left\{x_{n}\right\}$ be a sequence generated by the following algorithm:

$$
\left\{\begin{array}{l}
x_{0} \in C \quad \text { chosen arbitrary, } \quad C_{0}=C, \\
u_{n} \in C \quad \text { such that } \quad F\left(J u_{n}, J y\right)+\frac{1}{r_{n}}\left\langle u_{n}-x_{n}, J y-J u_{n}\right\rangle \geq 0, \quad \forall y \in C, \\
y_{n}=\alpha_{n} x_{n}+\left(1-\alpha_{n}\right) u_{n}, \\
C_{n+1}=\left\{z \in C_{n}: \phi\left(y_{n}, z\right) \leq \phi\left(x_{n}, z\right)\right\}, \\
x_{n+1}=R_{C_{n+1}}\left(x_{0}\right), \quad \forall n \in \mathbb{N} \cup\{0\},
\end{array}\right.
$$

where J is the duality mapping on $E$, the sequence $\left\{\alpha_{n}\right\} \subset[0,1]$ such that $\lim _{\sup _{n \rightarrow \infty}} \alpha_{n}<1$, $r_{n} \subset[a, \infty)$ for some $a>0$, and $R_{C_{n+1}}$ is the sunny generalized nonexpansive retraction from E onto $C_{n+1}$.

Then the sequence $\left\{x_{n}\right\}$ converges strongly to some point $p=R_{\mathrm{EP}(F)}\left(x_{0}\right)$, where $R_{\mathrm{EP}(F)}$ is the sunny generalized nonexpansive retraction from $E$ to $\mathrm{EP}(F)$.

In 2010, Plubtieng and Sriprad [8] proved the existence theorem of the variational inequality problem for skew monotone operator defined on the dual space of a smooth Banach space, and they established a weak convergence theorem for finding a solution of the 
variational inequality problem using the projection algorithm method with a new projection, which was introduced by Ibaraki and Takahashi [9] and Iiduka and Takahashi [10] in Banach spaces.

Theorem PS Let E be a uniformly convex and 2-uniformly smooth Banach space whose duality mapping $J$ is weakly sequentially continuous. Let $C$ be a nonempty, closed and convex subset of $E$ such that $J(C)$ is closed and convex, and let $A$ be an $\alpha$-inverse-strongly-skewmonotone operator of $J(C)$ into $E$ such that $\mathrm{VI}(J(C), A) \neq \emptyset$ and $\|A J z\| \leq\|A J y-A J z\|$, for all $y \in C$ and $z \in \operatorname{VI}(J(C), A)$. Let $\left\{x_{n}\right\}$ be a sequence defined by $x_{1}=x \in C$ and

$$
x_{n+1}=R_{C}\left(x_{n}-\lambda_{n} A J x_{n}\right)
$$

for every $n=1,2,3, \ldots$, where $R_{C}$ is the sunny generalized nonexpansive retraction of $E$ into $C,\left\{\alpha_{n}\right\} \subset[a, b]$, for some $a, b$ with $0<a<b<\frac{\alpha}{c}$, where $c>0$ is a constant satisfying $\|J x-J y\| \leq c\|x-y\|$ for all $x, y \in C$. Then the sequence $\left\{x_{n}\right\}$ converges weakly to some element $z \in \mathrm{VI}(J(C), A)$. Further $z=\lim _{n \rightarrow \infty} R_{\mathrm{VI}(J(C), A)}\left(x_{n}\right)$.

In this paper, motivated and inspired by the previously mentioned results, we study the existence theorem for a generalized equilibrium problem with a bifunction defined on the dual space of a Banach space, and we also construct an iterative procedure generated by a hybrid method for solving the solution of a generalized equilibrium problem by using the sunny generalized nonexpansive retraction. Under some suitable assumptions, the strong convergence theorems are established in Banach spaces. The results obtained in this paper extend and improve several recent results in this area.

\section{Preliminaries}

Definition 2.1 Let $C$ be a nonempty and closed subset of a smooth Banach space.

(1) A mapping $T: C \rightarrow C$ is said to be closed if for each $\left\{x_{n}\right\} \subset C, x_{n} \rightarrow x$ and $T x_{n} \rightarrow y$ imply that $T x=y$.

(2) A mapping $T: C \rightarrow C$ is said to be nonexpansive if

$$
\|T x-T y\| \leq\|x-y\|, \quad \forall x, y \in C .
$$

(3) A mapping $T: C \rightarrow C$ is said to be $\phi$-nonexpansive if $\operatorname{Fix}(T) \neq \emptyset$, and

$$
\phi(T x, T y) \leq \phi(x, y), \quad \forall x, y \in C .
$$

(4) A mapping $T: C \rightarrow C$ is said to be generalized nonexpansive [11] if $\operatorname{Fix}(T) \neq \emptyset$, and

$$
\phi(T x, p) \leq \phi(x, p), \quad \forall x \in C, p \in \operatorname{Fix}(T) .
$$

Definition 2.2 [11] Let $C$ be a nonempty and closed subset of a smooth Banach space $E$. A mapping $R: E \rightarrow C$ is called

(1) a retraction if $R^{2}=R$;

(2) a sunny if $R(R x+t(x-R x))=R x$ for all $x \in E$ and $t \geq 0$.

We also know that if $E$ is a smooth, strictly convex and reflexive Banach space, and $C$ is nonempty, closed and convex subset of $E$, then there exists a sunny generalized nonex- 
pansive retraction $R_{C}$ of $E$ onto $C$ if and only if $J(C)$ is closed and convex. In this case, $R_{C}$ is given by

$$
R_{C}=J^{-1} \Pi_{J(C)} J
$$

Definition 2.3 [11] Let $C$ be a nonempty and closed subset of a smooth Banach space $E$. The set $C$ is called a sunny generalized nonexpansive retraction of $E$ if there exists a sunny generalized nonexpansive $R$ from $E$ onto $C$.

Lemma 2.4 [9] Let $C$ be a nonempty and closed subset of a smooth and strictly convex Banach space $E$, and let $R$ be a retraction from $E$ onto $C$. Then the following are equivalent:

(1) $R$ is sunny generalized nonexpansive;

(2) $\langle x-R x, J y-J R x\rangle \leq 0$ for all $x \in E$ and $y \in C$.

Lemma 2.5 [9] Let C be a nonempty, closed and sunny generalized nonexpansive retraction of a smooth and strictly convex Banach space E. Then the sunny generalized nonexpansive retraction from $E$ onto $C$ is uniquely determined.

Lemma 2.6 [9] Let $C$ be a nonempty and closed subset of a smooth and strictly convex Banach space $E$ such that there exists a sunny generalized nonexpansive retraction $R$ from $E$ onto $C$. Let $x \in E$ and $z \in C$. Then the following hold:

(1) $z=R x$ if and only if $\langle x-z, J y-J z\rangle \leq 0$ for all $y \in C$;

(2) $\phi(x, R x)+\phi(R x, z) \leq \phi(x, z)$.

Lemma 2.7 [12] Let $C$ be a nonempty and closed subset of a smooth, strictly convex and reflexive Banach space $E$. Then the following are equivalent:

(1) $C$ is a sunny generalized nonexpansive retraction of $E$;

(2) $J(C)$ is closed and convex.

Remark 2.8 From Lemmas 2.5 and 2.7. If $E$ is a Hilbert space, then a sunny generalized nonexpansive retraction from $E$ onto $C$ reduces to a metric projection operator $P$ from $E$ onto $C$.

Lemma 2.9 [12] Let E be a smooth, strictly convex and reflexive Banach space, let $C$ be a nonempty, closed and sunny generalized nonexpansive retraction of $E$, and let $R$ be the sunny generalized nonexpansive retraction from $E$ onto $C$. Let $x \in E$ and $z \in C$. Then the following are equivalent:

(1) $z=R x$;

(2) $\phi(x, z)=\min _{y \in C} \phi(x, y)$.

Lemma 2.10 [7] Let E be a uniformly smooth and strictly convex real Banach space, and let $\left\{x_{n}\right\}$ and $\left\{y_{n}\right\}$ be two sequences of $E$. If $\phi\left(x_{n}, y_{n}\right) \rightarrow 0$ and either $\left\{x_{n}\right\}$ or $\left\{y_{n}\right\}$ is bounded, then $\left\|x_{n}-y_{n}\right\| \rightarrow 0$.

Lemma 2.11 [13] Let E be a uniformly smooth and strictly convex real Banach space with the Kadec-Klee property, and let $C$ be a nonempty, closed and convex subset of E. Let $\left\{x_{n}\right\}$ and $\left\{y_{n}\right\}$ be two sequences in $C$ and $p \in E$. If $x_{n} \rightarrow p$ and $\phi\left(x_{n}, y_{n}\right) \rightarrow 0$, then $y_{n} \rightarrow p$. 
Lemma 2.12 [14] Let $\left\{a_{n}\right\}$ and $\left\{b_{n}\right\}$ be two sequences of nonnegative real numbers satisfying the inequality

$$
a_{n+1} \leq a_{n}+b_{n}, \quad \forall n \geq 1 .
$$

If $\sum_{n=0}^{\infty} b_{n}<\infty$, then $\lim _{n \rightarrow \infty} a_{n}$ exists.

Now, let us recall the following well-known concept and the result.

Definition 2.13 [15] Let $B$ be a subset of a topological vector space $X$. A mapping $G$ : $B \rightarrow 2^{X}$ is called a KKM mapping if conv $\left\{x_{1}, x_{2}, x_{3}, \ldots, x_{m}\right\} \subset \bigcup_{i=1}^{m} G\left(x_{i}\right)$, for $x_{i} \in B$ and $i=1,2,3, \ldots, m$, where conv $A$ denotes the convex hull of the set $A$.

In [16], Ky Fan gave the following famous infinite-dimensional generalization of Knaster, Kuratowski and Mazurkiewicz's classical finite-dimensional result.

Lemma 2.14 [16] Let B be a subset of a Hausdorff topological vector space $X$, and let $G$ : $B \rightarrow 2^{X}$ be a KKM mapping. If $G(x)$ is closed for all $x \in B$ and is compact for at least one $x \in B$, then $\bigcap_{x \in B} G(x) \neq \emptyset$.

\section{Existence theorem}

In this section, we prove the existence theorem of a solution for a generalized equilibrium problem with a bifunction defined on the dual space of a Banach space. Now, we use the concept of KKM mapping to prove the lemma for our main result.

Lemma 3.1 Let $C$ be a nonempty, compact and convex subset of a uniformly smooth, strictly convex and reflexive Banach space $E$, and let $J$ be the duality mapping from $E$ into $E^{*}$ such that $J(C)$ is closed and convex, let us assume that a bifunction $F: J(C) \times J(C) \rightarrow \mathbb{R}$ satisfies the following conditions (DA1)-(DA4), let $C^{*}$ be a nonempty, closed and convex subset of $E^{*}$, and let $A: C^{*} \rightarrow E$ be an $\alpha$-inverse strongly skew monotone. Let any $r>0$ be a given real number, and let $x \in E$ be any point. Then there exists $z \in C$ such that

$$
F(J z, J y)+\langle A J z, J y-J z\rangle+\frac{1}{r}\langle z-x, J(y-z)\rangle \geq 0, \quad \forall y \in C .
$$

Proof Let $x_{0}$ be any point in $E$. For each $y \in C$, we define the mapping $H: C \rightarrow 2^{E}$ as follows:

$$
H(y)=\left\{z \in C: F(J z, J y)+\langle A J z, J y-J z\rangle+\frac{1}{r}\left\langle z-x_{0}, J(y-z)\right\rangle \geq 0, \forall y \in C\right\} .
$$

It is easy to see that $y \in H(y)$, and hence $H(y) \neq \emptyset$.

(a) First, we show that $H$ is a KKM mapping.

Suppose that $H$ is not a KKM mapping. Then there exists a finite subset $\left\{y_{1}, y_{2}, \ldots, y_{m}\right\}$ of $C$ and $\alpha_{i}>0$ with $\sum_{i=1}^{m} \alpha_{i}=1$ such that $\hat{x}=\sum_{i=1}^{m} \alpha_{i} y_{i} \notin \bigcup_{i=1}^{m} H\left(y_{i}\right)$ for all $i=1,2,3, \ldots, m$. It follows from the definition of a mapping $H$ that

$$
F\left(J \hat{x}, J y_{i}\right)+\left\langle A J \hat{x}, J y_{i}-J \hat{x}\right\rangle+\frac{1}{r}\left\langle\hat{x}-x_{0}, J\left(y_{i}-\hat{x}\right)\right\rangle<0 \quad \text { for all } i=1,2,3, \ldots, m .
$$


By the assumptions of (DA1) and (DA4), we get

$$
\begin{aligned}
0 & =F(J \hat{x}, J \hat{x})+\langle A J \hat{x}, J \hat{x}-J \hat{x}\rangle+\frac{1}{r}\left\langle\hat{x}-x_{0}, J(\hat{x}-\hat{x})\right\rangle \\
& \leq \sum_{i=1}^{m} \alpha_{i}\left(F\left(J \hat{x}, J y_{i}\right)+\left\langle A J \hat{x}, J y_{i}-J \hat{x}\right\rangle+\frac{1}{r}\left\langle\hat{x}-x_{0}, J\left(y_{i}-\hat{x}\right)\right\rangle\right) \\
& <0,
\end{aligned}
$$

which is a contradiction. Thus, $H$ is a KKM mapping on $C$.

(b) Next, we show that $H(y)$ is closed for all $y \in C$.

Let $\left\{z_{n}\right\}$ be a sequence in $H(y)$ such that $z_{n} \rightarrow z_{0}$, as $n \rightarrow \infty$.

It then follows from $z_{n} \in H(y)$ that

$$
F\left(J z_{n}, J y\right)+\left\langle A J z_{n}, J y-J z_{n}\right\rangle+\frac{1}{r}\left\langle z_{n}-x_{0}, J\left(y-z_{n}\right)\right\rangle \geq 0 .
$$

By assumption (DA3), the continuity of $J$ and the lower semicontinuity of $\|\cdot\|^{2}$, we obtain from (3.1) that

$$
\begin{aligned}
0 \leq & \liminf _{n \rightarrow \infty}\left(F\left(J z_{n}, J y\right)+\left\langle A J z_{n}, J y-J z_{n}\right\rangle+\frac{1}{r}\left\langle z_{n}-x_{0}, J\left(y-z_{n}\right)\right\rangle\right) \\
\leq & \limsup _{n \rightarrow \infty}\left(F\left(J z_{n}, J y\right)+\left\langle A J z_{n}, J y-J z_{n}\right\rangle+\frac{1}{r}\left\langle z_{n}-x_{0}, J\left(y-z_{n}\right)\right\rangle\right) \\
= & \limsup _{n \rightarrow \infty}\left(F\left(J z_{n}, J y\right)+\left\langle A J z_{n}, J y-J z_{n}\right\rangle+\frac{1}{r}\left\langle\left(z_{n}-y\right)+\left(y-x_{0}\right), J\left(y-z_{n}\right)\right\rangle\right) \\
= & \limsup _{n \rightarrow \infty}\left(F\left(J z_{n}, J y\right)+\left\langle A J z_{n}, J y-J z_{n}\right\rangle+\frac{1}{r}\left\langle z_{n}-y, J\left(y-z_{n}\right)\right\rangle+\frac{1}{r}\left\langle y-x_{0}, J\left(y-z_{n}\right)\right\rangle\right) \\
= & \limsup _{n \rightarrow \infty}\left(F\left(J z_{n}, J y\right)+\left\langle A J z_{n}, J y-J z_{n}\right\rangle+\frac{1}{r}\left\langle y-x_{0}, J\left(y-z_{n}\right)\right\rangle-\frac{1}{r}\left\langle y-z_{n}, J\left(y-z_{n}\right)\right\rangle\right) \\
= & \limsup _{n \rightarrow \infty}\left(F\left(J z_{n}, J y\right)+\left\langle A J z_{n}, J y-J z_{n}\right\rangle+\frac{1}{r}\left\langle y-x_{0}, J\left(y-z_{n}\right)\right\rangle-\frac{1}{r}\left\|y-z_{n}\right\|^{2}\right) \\
\leq & \limsup _{n \rightarrow \infty} F\left(J z_{n}, J y\right)+\limsup \left\langle A J z_{n}, J y-J z_{n}\right\rangle \\
& +\frac{1}{r} \limsup _{n \rightarrow \infty}\left\langle y-x_{0}, J\left(y-z_{n}\right)\right\rangle-\liminf _{n \rightarrow \infty} \frac{1}{r}\left\|y-z_{n}\right\|^{2} \\
= & F\left(J z_{0}, J y\right)+\left\langle A J z_{0}, J y-J z_{0}\right\rangle+\frac{1}{r}\left\langle\left(y-x_{0}\right)+\left(z_{0}-y\right), J\left(y-z_{0}\right)\right\rangle \\
\leq & F\left(J z_{0}, J y\right)+\left\langle A J z_{0}, J y-J z_{0}\right\rangle+\frac{1}{r}\left\langle y-x_{0}, J\left(y-z_{0}\right)\right\rangle-\frac{1}{r}\left\|y-z_{0}\right\|^{2} \\
= & F\left(J z_{0}, J y\right)+\left\langle A J z_{0}, J y-J z_{0}\right\rangle+\frac{1}{r}\left\langle y-x_{0}, J\left(y-z_{0}\right)\right\rangle-\frac{1}{r}\left\langle y-z_{0}, J\left(y-z_{0}\right)\right\rangle \\
& F\left(J z_{0}, J y\right)+\left\langle A J z_{0}, J y-J z_{0}\right\rangle+\frac{1}{r}\left\langle z_{0}-x_{0}, J\left(y-z_{0}\right)\right\rangle . \\
& \\
&
\end{aligned}
$$


Now, we get

$$
F\left(J z_{0}, J y\right)+\left\langle A J z_{0}, J y-J z_{0}\right\rangle+\frac{1}{r}\left\langle z_{0}-x_{0}, J\left(y-z_{0}\right)\right\rangle \geq 0 .
$$

Therefore, $z_{0} \in H(y)$, and so $H(y)$ is closed for all $y \in C$.

(c) We show that $H(y)$ is weakly compact.

Now, we know that $H(y)$ is closed and subset of $C$.

Since $C$ is compact. Therefore, $H(y)$ is compact, and then $H(y)$ is weakly compact.

By using (a), (b), (c) and Lemma 2.14, we can conclude that $\bigcap_{y \in C} H(y) \neq \emptyset$.

Therefore, there exists $z \in C$ such that

$$
F(J z, J y)+\langle A J z, J y-J z\rangle+\frac{1}{r}\langle z-x, J(y-z)\rangle \geq 0, \quad \forall y \in C
$$

Theorem 3.2 Let C be a nonempty, closed and convex subset of a uniformly smooth, strictly convex and reflexive Banach space $E$, and let $J$ be the duality mapping from $E$ into $E^{*}$ such that $J(C)$ is closed and convex, let us assume that a bifunction $F: J(C) \times J(C) \rightarrow \mathbb{R}$ satisfies the following conditions (DA1)-(DA4), let $C^{*}$ be a nonempty, closed and convex subset of $E^{*}$, and let $A: C^{*} \rightarrow E$ be an $\alpha$-inverse strongly skew monotone and hemicontinuous. Let any $r>0$ be a given real number, and let $x \in E$ be any point. We define a mapping $T_{r}: E \rightarrow C$ as follows:

$$
T_{r}(x)=\left\{z \in C: F(J z, J y)+\langle A J z, J y-J z\rangle+\frac{1}{r}\langle z-x, J(y-z)\rangle \geq 0, \forall y \in C\right\} .
$$

Then the following conclusions hold:

(1) $T_{r}$ is single-valued;

(2) $\left\langle T_{r} x-T_{r} y, J\left(T_{r} x-T_{r} y\right)\right\rangle \leq\left\langle x-y, J\left(T_{r} x-T_{r} y\right)\right\rangle, \forall x, y \in E$;

(3) $\operatorname{Fix}\left(T_{r}\right)=\operatorname{GEP}(F, A)$;

(4) $J(\operatorname{GEP}(F, A))$ is closed and convex.

Proof We complete this proof by four items below.

(1) We show that $T_{r}$ is single-valued.

From the definition of $T_{r}(x)$, it is easy to see that

$$
F(J y, J y)+\langle A J y, J y-J y\rangle+\frac{1}{r}\langle z-y, J(y-y)\rangle=0 \geq 0 .
$$

Therefore, $y \in T_{r}(x)$. Hence, $T_{r}(x) \neq \emptyset$.

Indeed, for any $x \in E$ and $r>0$, let $z_{1}, z_{2} \in T_{r}(x)$. Then

$$
F\left(J z_{1}, J z_{2}\right)+\left\langle A J z_{1}, J z_{2}-J z_{1}\right\rangle+\frac{1}{r}\left\langle z_{1}-x, J\left(z_{2}-z_{1}\right)\right\rangle \geq 0
$$

and

$$
F\left(J z_{2}, J z_{1}\right)+\left\langle A J z_{2}, J z_{1}-J z_{2}\right\rangle+\frac{1}{r}\left\langle z_{2}-x, J\left(z_{1}-z_{2}\right)\right\rangle \geq 0 .
$$


Adding the two inequalities, we have

$$
\begin{aligned}
0 \leq & F\left(J z_{1}, J z_{2}\right)+F\left(J z_{2}, J z_{1}\right)+\left\langle A J z_{1}, J z_{2}-J z_{1}\right\rangle+\left\langle A J z_{2}, J z_{1}-J z_{2}\right\rangle \\
& +\frac{1}{r}\left\langle z_{1}-x, J\left(z_{2}-z_{1}\right)\right\rangle+\frac{1}{r}\left\langle z_{2}-x, J\left(z_{1}-z_{2}\right)\right\rangle \\
= & F\left(J z_{1}, J z_{2}\right)+F\left(J z_{2}, J z_{1}\right)+\left\langle A J z_{1}, J z_{2}-J z_{1}\right\rangle-\left\langle A J z_{2}, J z_{2}-J z_{1}\right\rangle \\
& +\frac{1}{r}\left\langle z_{1}-x, J\left(z_{2}-z_{1}\right)\right\rangle-\frac{1}{r}\left\langle z_{2}-x, J\left(z_{2}-z_{1}\right)\right\rangle \\
= & F\left(J z_{1}, J z_{2}\right)+F\left(J z_{2}, J z_{1}\right)+\left\langle A J z_{1}-A J z_{2}, J z_{2}-J z_{1}\right\rangle+\frac{1}{r}\left\langle\left(z_{1}-x\right)-\left(z_{2}-x\right), J\left(z_{2}-z_{1}\right)\right\rangle \\
= & F\left(J z_{1}, J z_{2}\right)+F\left(J z_{2}, J z_{1}\right)+\left\langle A J z_{1}-A J z_{2}, J z_{2}-J z_{1}\right\rangle+\frac{1}{r}\left\langle z_{1}-z_{2}, J\left(z_{2}-z_{1}\right)\right\rangle \\
= & F\left(J z_{1}, J z_{2}\right)+F\left(J z_{2}, J z_{1}\right)-\left\langle A J z_{1}-A J z_{2}, J z_{1}-J z_{2}\right\rangle+\frac{1}{r}\left\langle z_{1}-z_{2}, J\left(z_{2}-z_{1}\right)\right\rangle .
\end{aligned}
$$

Therefore, we obtain

$$
F\left(J z_{1}, J z_{2}\right)+F\left(J z_{2}, J z_{1}\right)-\left\langle A J z_{1}-A J z_{2}, J z_{1}-J z_{2}\right\rangle+\frac{1}{r}\left\langle z_{1}-z_{2}, J\left(z_{2}-z_{1}\right)\right\rangle \geq 0 .
$$

From condition (DA2) and the fact that $A$ is an $\alpha$-inverse strongly skew monotone, we have

$$
\begin{aligned}
0 & \leq F\left(J z_{1}, J z_{2}\right)+F\left(J z_{2}, J z_{1}\right)-\left\langle A J z_{1}-A J z_{2}, J z_{1}-J z_{2}\right\rangle+\frac{1}{r}\left\langle z_{1}-z_{2}, J\left(z_{2}-z_{1}\right)\right\rangle \\
& \leq-\alpha\left\|A J z_{1}-A J z_{2}\right\|^{2}+\frac{1}{r}\left\langle z_{1}-z_{2}, J\left(z_{2}-z_{1}\right)\right\rangle \\
& \leq \frac{1}{r}\left\langle z_{1}-z_{2}, J\left(z_{2}-z_{1}\right)\right\rangle .
\end{aligned}
$$

Since $r>0, J$ is monotone, and $E$ is strictly convex, we obtain

$$
z_{1}=z_{2}
$$

This implies that $T_{r}$ is single-valued.

(2) We show that $\left\langle T_{r} x-T_{r} y, J\left(T_{r} x-T_{r} y\right)\right\rangle \leq\left\langle x-y, J\left(T_{r} x-T_{r} y\right)\right\rangle$ for all $x, y \in E$. Indeed, for any $x, y \in C$ and $r>0$, we have

$$
F\left(J T_{r} x, J T_{r} y\right)+\left\langle A J T_{r} x, J T_{r} y-J T_{r} x\right\rangle+\frac{1}{r}\left\langle T_{r} x-x, J\left(T_{r} y-T_{r} x\right)\right\rangle \geq 0,
$$

and

$$
F\left(J T_{r} y, J T_{r} x\right)+\left\langle A J T_{r} y, J T_{r} x-J T_{r} y\right\rangle+\frac{1}{r}\left\langle T_{r} y-y, J\left(T_{r} x-T_{r} y\right)\right\rangle \geq 0 .
$$

Adding the two inequalities, we have

$$
\begin{aligned}
0 \leq & F\left(J T_{r} x, J T_{r} y\right)+F\left(J T_{r} y, J T_{r} x\right)+\left\langle A J T_{r} x, J T_{r} y-J T_{r} x\right\rangle+\left\langle A J T_{r} y, J T_{r} x-J T_{r} y\right\rangle \\
& +\frac{1}{r}\left\langle T_{r} x-x, J\left(T_{r} y-T_{r} x\right)\right\rangle+\frac{1}{r}\left\langle T_{r} y-y, J\left(T_{r} x-T_{r} y\right)\right\rangle \\
= & F\left(J T_{r} x, J T_{r} y\right)+F\left(J T_{r} y, J T_{r} x\right)+\left\langle A J T_{r} x, J T_{r} y-J T_{r} x\right\rangle-\left\langle A J T_{r} y, J T_{r} y-J T_{r} x\right\rangle
\end{aligned}
$$




$$
\begin{aligned}
& +\frac{1}{r}\left\langle T_{r} x-x, J\left(T_{r} y-T_{r} x\right)\right\rangle-\frac{1}{r}\left\langle T_{r} y-y, J\left(T_{r} y-T_{r} x\right)\right\rangle \\
= & F\left(J T_{r} x, J T_{r} y\right)+F\left(J T_{r} y, J T_{r} x\right)+\left\langle A J T_{r} x-A J T_{r} y, J T_{r} y-J T_{r} x\right\rangle \\
& +\frac{1}{r}\left\langle\left(T_{r} x-x\right)-\left(T_{r} y-y\right), J\left(T_{r} y-T_{r} x\right)\right\rangle \\
= & F\left(J T_{r} x, J T_{r} y\right)+F\left(J T_{r} y, J T_{r} x\right)+\left\langle A J T_{r} x-A J T_{r} y, J T_{r} y-J T_{r} x\right\rangle \\
& +\frac{1}{r}\left\langle\left(T_{r} x-T_{r} y\right)-(x-y), J\left(T_{r} y-T_{r} x\right)\right\rangle \\
= & F\left(J T_{r} x, J T_{r} y\right)+F\left(J T_{r} y, J T_{r} x\right)-\left\langle A J T_{r} x-A J T_{r} y, J T_{r} x-J T_{r} y\right\rangle \\
& +\frac{1}{r}\left\langle\left(T_{r} x-T_{r} y\right)-(x-y), J\left(T_{r} y-T_{r} x\right)\right\rangle .
\end{aligned}
$$

It follows that

$$
\begin{gathered}
F\left(J T_{r} x, J T_{r} y\right)+F\left(J T_{r} y, J T_{r} x\right)-\left\langle A J T_{r} x-A J T_{r} y, J T_{r} x-J T_{r} y\right\rangle \\
\quad+\frac{1}{r}\left\langle\left(T_{r} x-T_{r} y\right)-(x-y), J\left(T_{r} y-T_{r} x\right)\right\rangle \geq 0 .
\end{gathered}
$$

From condition (DA2) and the fact that $A$ is an $\alpha$-inverse strongly skew monotone, we get

$$
\begin{aligned}
0 \leq & F\left(J T_{r} x, J T_{r} y\right)+F\left(J T_{r} y, J T_{r} x\right)-\left\langle A J T_{r} x-A J T_{r} y, J T_{r} x-J T_{r} y\right\rangle \\
& +\frac{1}{r}\left\langle\left(T_{r} x-T_{r} y\right)-(x-y), J\left(T_{r} y-T_{r} x\right)\right\rangle \\
\leq & -\alpha\left\|A J T_{r} x-A J T_{r} y\right\|^{2}+\frac{1}{r}\left\langle\left(T_{r} x-T_{r} y\right)-(x-y), J\left(T_{r} y-T_{r} x\right)\right\rangle \\
\leq & \frac{1}{r}\left\langle\left(T_{r} x-T_{r} y\right)-(x-y), J\left(T_{r} y-T_{r} x\right)\right\rangle \\
\leq & -\frac{1}{r}\left\langle\left(T_{r} x-T_{r} y\right)-(x-y), J\left(T_{r} x-T_{r} y\right)\right\rangle .
\end{aligned}
$$

Since $r>0$, we have

$$
\left\langle\left(T_{r} x-T_{r} y\right)-(x-y), J\left(T_{r} x-T_{r} y\right)\right\rangle \leq 0 .
$$

Therefore, we also have

$$
\left\langle T_{r} x-T_{r} y, J\left(T_{r} x-T_{r} y\right)\right\rangle-\left\langle x-y, J\left(T_{r} x-T_{r} y\right)\right\rangle \leq 0 .
$$

This implies that

$$
\left\langle T_{r} x-T_{r} y, J\left(T_{r} x-T_{r} y\right)\right\rangle \leq\left\langle x-y, J\left(T_{r} x-T_{r} y\right)\right\rangle .
$$

(3) We show that $\operatorname{Fix}\left(T_{r}\right)=\operatorname{GEP}(F, A)$.

It is easy to see that

$$
\begin{aligned}
z \in \operatorname{Fix}\left(T_{r}\right) & \Leftrightarrow \quad z=T_{r} z \\
& \Leftrightarrow \quad F(J z, J y)+\langle A J z, J y-J z\rangle+\frac{1}{r}\langle z-z, J(y-z)\rangle \geq 0
\end{aligned}
$$




$$
\begin{aligned}
& \Leftrightarrow \quad F(J z, J y)+\langle A J z, J y-J z\rangle \geq 0 \\
& \Leftrightarrow \quad z \in \operatorname{GEP}(F, A) .
\end{aligned}
$$

This implies that $\operatorname{Fix}\left(T_{r}\right)=\operatorname{GEP}(F, A)$.

(4) We show that $J(\operatorname{GEP}(F, A))$ is closed and convex.

For each $y \in C$, we define the mapping $G: C \rightarrow 2^{E}$ as follows:

$$
G(y)=\{z \in C: F(J z, J y)+\langle A J z, J y-J z\rangle \geq 0\}
$$

It is easy to see that $y \in G(y)$, so that $G(y) \neq \emptyset$.

Next, we show that $G$ is a KKM mapping.

Suppose that $G$ is not a KKM mapping. Then there exists a finite subset $\left\{z_{1}, z_{2}, \ldots, z_{m}\right\}$ of $C$, and $\beta_{i}>0$ with $\sum_{i=1}^{m} \beta_{i}=1$ such that $\hat{z}=\sum_{i=1}^{m} \beta_{i} z_{i} \notin G\left(z_{i}\right)$ for all $i=1,2,3, \ldots, m$. Then we have

$$
F\left(J \hat{z}, J z_{i}\right)+\left\langle A J \hat{z}, J z_{i}-J \hat{z}\right\rangle<0, \quad i=1,2,3, \ldots, m
$$

It follows from (DA1) and (DA4) that

$$
0=F(J \hat{z}, J \hat{z})+\langle A J \hat{z}, J \hat{z}-J \hat{z}\rangle \leq \sum_{i=1}^{m} \beta_{i}\left(F\left(J \hat{z}, J z_{i}\right)+\left\langle A J \hat{z}, J z_{i}-J \hat{z}\right\rangle\right)<0
$$

which is the contradiction. Hence, $G$ is a KKM mapping on $C$.

(4.1) Next, we show that $G(y)$ is closed for each $y \in C$.

For any $y \in C$, let $\left\{z_{n}\right\}$ be any sequence in $G(y)$ such that $z_{n} \rightarrow z_{0}$, as $n \rightarrow \infty$.

Hence, $z_{n}-x_{0} \rightarrow z_{0}-x_{0}$, as $n \rightarrow \infty$.

Next, we show that $z_{0} \in G(y)$. Then for each $y \in C$, we have

$$
F\left(J z_{n}, J y\right)+\left\langle A J z_{n}, J y-J z_{n}\right\rangle \geq 0
$$

It follows from assumption (DA3) that

$$
F\left(J z_{0}, J y\right)+\left\langle A J z_{0}, J y-J z_{0}\right\rangle \geq \limsup _{n \rightarrow \infty} F\left(J z_{n}, J y\right)+\lim _{n \rightarrow \infty}\left\langle A J z_{n}, J y-J z_{n}\right\rangle \geq 0 .
$$

This implies that $z_{0} \in G(y)$, and hence $G(y)$ is closed for each $y \in C$.

Since $J$ is continuous. Therefore, $\bigcap_{y \in C} G(y)=J(\operatorname{GEP}(F, A))$ is closed.

(4.2) Next, we show that $J(\operatorname{GEP}(F, A))$ is convex.

Let $z_{1}^{*}, z_{2}^{*} \in J(\operatorname{GEP}(F, A))$, then we have $z_{1}^{*}=J z_{1} \in J(C)$ and $z_{2}^{*}=J z_{2} \in J(C)$, where $z_{1}, z_{2} \in C$.

For $k, t \in(0,1)$, let $z^{*}=k z_{1}^{*}+(1-k) z_{2}^{*}$ and for any $y \in C$, we set $x_{t}^{*}=t J y+(1-t) z^{*}$.

It follows from (DA1) and (DA4) that

$$
\begin{aligned}
0 & =F\left(x_{t}^{*}, x_{t}^{*}\right) \\
& \leq F\left(x_{t}^{*}, t J y+(1-t) z^{*}\right) \\
& \leq t F\left(x_{t}^{*}, J y\right)+(1-t) F\left(x_{t}^{*}, z^{*}\right) \\
& \leq t F\left(x_{t}^{*}, J y\right)
\end{aligned}
$$


and

$$
\begin{aligned}
0 & =\left\langle A x_{t}^{*}, x_{t}^{*}-x_{t}^{*}\right\rangle \\
& =\left\langle A x_{t}^{*},\left(x_{t}^{*}-J y\right)+\left(J y-x_{t}^{*}\right)\right\rangle \\
& =\left\langle A x_{t}^{*}, x_{t}^{*}-J y\right\rangle+\left\langle A x_{t}^{*}, J y-x_{t}^{*}\right\rangle \\
& =\left\langle A x_{t}^{*}, x_{t}^{*}-J y\right\rangle-\left\langle A x_{t}^{*}, x_{t}^{*}-J y\right\rangle \\
& \leq\left\langle A x_{t}^{*}, x_{t}^{*}-J y\right\rangle \\
& =\left\langle A x_{t}^{*}, t J y+(1-t) z^{*}-J y\right\rangle \\
& =\left\langle A x_{t}^{*}, t J y+(1-t) z^{*}-(t+(1-t)) J y\right\rangle \\
& =\left\langle A x_{t}^{*},(1-t)\left(z^{*}-J y\right)\right\rangle \\
& =(1-t)\left\langle A x_{t}^{*}, z^{*}-J y\right\rangle \\
& =(t-1)\left\langle A x_{t}^{*}, J y-z^{*}\right\rangle \\
& \leq t\left\langle A x_{t}^{*}, J y-z^{*}\right\rangle .
\end{aligned}
$$

Adding two inequalities (3.7) and (3.8) and dividing by $t>0$, we get

$$
\begin{aligned}
0 & \leq t F\left(x_{t}^{*}, J y\right)+t\left\langle A x_{t}^{*}, J y-z^{*}\right\rangle \\
& \leq F\left(x_{t}^{*}, J y\right)+\left\langle A x_{t}^{*}, J y-z^{*}\right\rangle .
\end{aligned}
$$

Letting $t$ to 0 by (DA3) and the hemicontinuous of $A$, we obtain

$$
F\left(z^{*}, J y\right)+\left\langle A z^{*}, J y-z^{*}\right\rangle \geq 0 \quad \text { for all } y \in C .
$$

Hence, $z^{*} \in J(\operatorname{GEP}(F, A))$, and thus, $J(\operatorname{GEP}(F, A))$ is convex.

This completes the proof.

\section{Convergence theorem}

In this section, we use the hybrid projection method for finding a solution of a generalized equilibrium problem in the dual space of Banach spaces.

Theorem 4.1 Let E be a uniformly smooth, strictly convex and reflexive real Banach space, which has a Kadec-Klee property, let $C$ be a nonempty, closed and convex subset of $E$, and let $J$ be the duality mapping from $E$ into $E^{*}$ such that $J(C)$ is closed and convex of $E^{*}$, let us assume that a bifunction $F: J(C) \times J(C) \rightarrow \mathbb{R}$ satisfies the following conditions (DA1)-(DA4), let $C^{*}$ be a nonempty, closed and convex subset of $E^{*}$, and let $A: C^{*} \rightarrow E$ be an $\alpha$-inverse strongly skew monotone. For $x \in E$, we define a mapping $T_{r_{n}}: E \rightarrow C$ as follows:

$$
T_{r_{n}}(x)=\left\{z \in C: F(J z, J y)+\langle A J z, J y-J z\rangle+\frac{1}{r_{n}}\langle z-x, J(y-z)\rangle \geq 0, \forall y \in C\right\} .
$$


Suppose that $\operatorname{GEP}(F, A) \neq \emptyset$. Let $\left\{x_{n}\right\}$ be a sequence generated by

$$
\left\{\begin{array}{l}
x_{0} \in C \quad \text { chosen arbitrary, } \quad C_{0}=C, \\
u_{n}=T_{r_{n}} x_{n} \\
y_{n}=\alpha_{n} x_{n}+\left(1-\alpha_{n}\right) u_{n}, \\
C_{n+1}=\left\{z \in C_{n}: \phi\left(y_{n}, z\right) \leq \phi\left(x_{n}, z\right)\right\} \\
x_{n+1}=R_{C_{n+1}}\left(x_{0}\right), \quad \forall n \geq 0
\end{array}\right.
$$

where $J$ is the duality mapping on $E,\left\{\alpha_{n}\right\}$ is the sequence in $[0,1]$ such that $\lim _{\sup } \rightarrow \infty \alpha_{n}<$ $1,\left\{r_{n}\right\} \subset[a, \infty)$ for some $a>0$ and $R_{C_{n+1}}$ is the sunny generalized nonexpansive retraction from $E$ onto $C_{n+1}$. Then the sequence $\left\{x_{n}\right\}$ converges strongly to $R_{\mathrm{GEP}(F, A)}\left(x_{0}\right)$, where $R_{\mathrm{GEP}(F, A)}$ is the sunny generalized nonexpansive retraction from $E$ onto $\operatorname{GEP}(F, A)$.

Proof We complete this proof by seven steps below.

Step 1. We show that $J\left(C_{n}\right)$ are closed and convex subsets of $E$ for each $n \in \mathbb{N} \cup\{0\}$.

It is obvious that $J\left(C_{0}\right)$ is closed and convex. Suppose that $J\left(C_{k}\right)$ is closed and convex for some $k \in \mathbb{N} \cup\{0\}$.

For each $z \in C_{n}$, we see that

$$
\begin{aligned}
\phi\left(y_{n}, z\right) \leq \phi\left(x_{n}, z\right) & \Leftrightarrow \quad\left\|y_{n}\right\|^{2}-2\left\langle y_{n}, J z\right\rangle+\|z\|^{2} \leq\left\|x_{n}\right\|^{2}-2\left\langle x_{n}, J z\right\rangle+\|z\|^{2} \\
& \Leftrightarrow \quad\left\|y_{n}\right\|^{2}-\left\|x_{n}\right\|^{2}-2\left\langle y_{n}, J z\right\rangle+2\left\langle x_{n}, J z\right\rangle \leq 0 \\
& \Leftrightarrow \quad\left\|y_{n}\right\|^{2}-\left\|x_{n}\right\|^{2}-2\left\langle y_{n}-x_{n}, J z\right\rangle \leq 0 .
\end{aligned}
$$

Hence, $J\left(C_{k+1}\right)$ is closed and convex. Therefore, $J\left(C_{n}\right)$ are closed and convex subsets of $E$ for each $n \in \mathbb{N} \cup\{0\}$.

Step 2. We show that $\operatorname{GEP}(F, A) \subset C_{n}$ for all $n \in \mathbb{N} \cup\{0\}$.

Note that $u_{n}=T_{r_{n}} x_{n}$ and $T_{r_{n}}$ is generalized nonexpansive.

From $C_{0}=C$, we have $\operatorname{GEP}(F, A) \subset C_{0}$. Suppose that $\operatorname{GEP}(F, A) \subset C_{k}$, for some $k \in$ $\mathbb{N} \cup\{0\}$.

For any $p \in \operatorname{GEP}(F, A)$, from algorithm (4.2) and the fact that $T_{r_{k}}$ is generalized nonexpansive, we compute

$$
\begin{aligned}
\phi\left(y_{k}, p\right)= & \phi\left(\alpha_{n} x_{k}+\left(1-\alpha_{n}\right) u_{k}, p\right) \\
= & \left\|\alpha_{k} x_{k}+\left(1-\alpha_{k}\right) u_{k}\right\|^{2}-2\left\langle\alpha_{k} x_{k}+\left(1-\alpha_{k}\right) u_{k}, J p\right\rangle+\|p\|^{2} \\
\leq & \alpha_{k}\left\|x_{k}\right\|^{2}+\left(1-\alpha_{k}\right)\left\|u_{k}\right\|^{2}-2 \alpha_{k}\left\langle x_{k}, J p\right\rangle-2\left(1-\alpha_{k}\right)\left\langle u_{k}, J p\right\rangle+\|p\|^{2} \\
= & \alpha_{k}\left\|x_{k}\right\|^{2}+\left(1-\alpha_{k}\right)\left\|u_{k}\right\|^{2}-2 \alpha_{k}\left\langle x_{k}, J p\right\rangle \\
& -2\left(1-\alpha_{k}\right)\left\langle u_{k}, J p\right\rangle+\left(\alpha_{k}+\left(1-\alpha_{k}\right)\right)\|p\|^{2} \\
= & \alpha_{k}\left\|x_{k}\right\|^{2}-2 \alpha_{k}\left\langle x_{k}, J p\right\rangle+\alpha_{k}\|p\|^{2}+\left(1-\alpha_{k}\right)\left\|u_{k}\right\|^{2} \\
& -2\left(1-\alpha_{k}\right)\left\langle u_{k}, J p\right\rangle+\left(1-\alpha_{k}\right)\|p\|^{2} \\
= & \alpha_{k} \phi\left(x_{k}, p\right)+\left(1-\alpha_{k}\right) \phi\left(u_{k}, p\right) \\
= & \alpha_{k} \phi\left(x_{k}, p\right)+\left(1-\alpha_{k}\right) \phi\left(T_{r_{k}} x_{k}, p\right)
\end{aligned}
$$




$$
\begin{aligned}
& \leq \alpha_{k} \phi\left(x_{k}, p\right)+\left(1-\alpha_{k}\right) \phi\left(x_{k}, p\right) \\
& =\phi\left(x_{k}, p\right)
\end{aligned}
$$

Therefore, $p \in C_{k}$. Hence, we get $p \in C_{k+1}$.

This implies that $\operatorname{GEP}(F, A) \subset C_{n}, \forall n \in \mathbb{N} \cup\{0\}$, and also the sequence $\left\{x_{n}\right\}$ is well defined.

Step 3. We show that $\left\{x_{n}\right\}$ is bounded.

From the definition of $\left\{x_{n}\right\}$, we know that $x_{n}=R_{C_{n}}\left(x_{0}\right)$ and $x_{n+1}=R_{C_{n+1}}\left(x_{0}\right) \in C_{n+1} \subset C_{n}$. For all $p \in \operatorname{GEP}(F, A) \subset C_{n}$, we have

$$
\begin{aligned}
\phi\left(x_{n}, x_{0}\right) & =\phi\left(R_{C_{n}}\left(x_{0}\right), x_{0}\right) \\
& \leq \phi\left(p, x_{0}\right)-\phi\left(p, R_{C_{n}}\left(x_{0}\right)\right) \\
& \leq \phi\left(p, x_{0}\right) .
\end{aligned}
$$

Then $\phi\left(x_{0}, x_{n}\right)$ is bounded. Therefore, $\left\{x_{n}\right\}$ is bounded, and also $\left\{T_{r_{n}} x_{n}\right\}$ is bounded.

Step 4. We show that there exists $\hat{p} \in C$ such that $x_{n} \rightarrow \hat{p}$, as $n \rightarrow \infty$.

Since $x_{n}=R_{C_{n}}\left(x_{0}\right)$ and $x_{n+1}=R_{C_{n+1}}\left(x_{0}\right) \in C_{n+1} \subset C_{n}$, we have

$$
\phi\left(x_{n}, x_{0}\right) \leq \phi\left(x_{n+1}, x_{0}\right), \quad \forall n \in \mathbb{N} \cup\{0\} .
$$

Therefore, the sequence $\left\{\phi\left(x_{n}, x_{0}\right)\right\}$ is nondecreasing. Hence, $\lim _{n \rightarrow \infty} \phi\left(x_{n}, x_{0}\right)$ exists.

By the definition of $C_{n}$, one has that $C_{m} \subset C_{n}$ and $x_{m}=R_{C_{m}}\left(x_{0}\right) \in C_{n}$ for any positive integer $m \geq n$. It follows that

$$
\begin{aligned}
\phi\left(x_{m}, x_{n}\right) & =\phi\left(x_{m}, R_{C_{n}}\left(x_{0}\right)\right) \\
& \leq \phi\left(x_{m}, x_{0}\right)-\phi\left(R_{C_{n}}\left(x_{0}\right), x_{0}\right) \\
& \leq \phi\left(x_{m}, x_{0}\right)-\phi\left(x_{n}, x_{0}\right) \\
& \rightarrow 0, \quad \text { as } m, n \rightarrow \infty .
\end{aligned}
$$

It follows from Lemma 2.10 that $\left\|x_{m}-x_{n}\right\| \rightarrow 0$, as $m, n \rightarrow \infty$.

Thus, the sequence $\left\{x_{n}\right\}$ is a Cauchy sequence.

Without loss of generality, we can assume that $x_{n} \rightarrow \hat{p}$. Since $\left\{x_{n}\right\}$ is bounded, and $E$ is reflexive.

We know that $C_{n+1} \subset C_{n}$, and $C_{n}$ is closed and convex, for $\hat{p} \in C_{n}$, we have

$$
\begin{aligned}
\liminf _{n \rightarrow \infty} \phi\left(x_{n}, x_{0}\right) & =\liminf _{n \rightarrow \infty}\left(\left\|x_{n}\right\|^{2}-2\left\langle x_{n}, J x_{0}\right\rangle+\left\|x_{0}\right\|^{2}\right) \\
& \geq\|\hat{p}\|^{2}-2\left\langle\hat{p}, J x_{0}\right\rangle+\left\|x_{0}\right\|^{2} \\
& =\phi\left(\hat{p}, x_{0}\right) .
\end{aligned}
$$

It follows that

$$
\phi\left(\hat{p}, x_{0}\right) \leq \liminf _{n \rightarrow \infty} \phi\left(x_{n}, x_{0}\right) \leq \phi\left(\hat{p}, x_{0}\right)
$$


This implies that

$$
\lim _{n \rightarrow \infty} \phi\left(x_{n}, x_{0}\right)=\phi\left(\hat{p}, x_{0}\right)
$$

So $\left\|x_{n}\right\| \rightarrow\|\hat{p}\|$. Since $x_{n} \rightarrow \hat{p}$. By the Kadec-Klee property of $E$, we obtain that

$$
x_{n} \rightarrow \hat{p}, \quad \text { as } n \rightarrow \infty \text {. }
$$

From $J$ is uniformly norm-to-norm continuous on bounded subset of $E$, we also have

$$
J x_{n} \rightarrow J \hat{p}, \quad \text { as } n \rightarrow \infty \text {. }
$$

Step 5. We show that $\left\|x_{n}-y_{n}\right\| \rightarrow 0$ and $\left\|x_{n}-u_{n}\right\| \rightarrow 0$, as $n \rightarrow \infty$.

Since $x_{n}=R_{C_{n}}\left(x_{0}\right)$ and $\lim _{n \rightarrow \infty} \phi\left(x_{n}, x_{0}\right)$ exists. We get

$$
\begin{aligned}
\phi\left(x_{n}, x_{n+1}\right) & =\phi\left(R_{C_{n}}\left(x_{0}\right), x_{n+1}\right) \\
& \leq \phi\left(x_{0}, x_{n+1}\right)-\phi\left(x_{0}, R_{C_{n}}\left(x_{0}\right)\right) \\
& =\phi\left(x_{0}, x_{n+1}\right)-\phi\left(x_{0}, x_{n}\right) \\
& \rightarrow 0, \quad \text { as } n \rightarrow \infty .
\end{aligned}
$$

From $x_{n+1}=R_{C_{n+1}}\left(x_{0}\right) \in C_{n+1} \subset C_{n}$, we have

$$
\phi\left(y_{n}, x_{n+1}\right) \leq \phi\left(x_{n}, x_{n+1}\right) \rightarrow 0, \quad \text { as } n \rightarrow \infty
$$

By Lemma 2.10, we obtain

$$
\left\|x_{n}-x_{n+1}\right\| \rightarrow 0 \quad \text { and } \quad\left\|y_{n}-x_{n+1}\right\| \rightarrow 0, \quad \text { as } n \rightarrow \infty
$$

Therefore,

$$
\left\|x_{n}-y_{n}\right\| \rightarrow 0, \quad \text { as } n \rightarrow \infty
$$

Since

$$
\begin{aligned}
0 & =\lim _{n \rightarrow \infty}\left\|x_{n}-y_{n}\right\| \\
& =\lim _{n \rightarrow \infty}\left\|x_{n}-\left(\alpha_{n} x_{n}+\left(1-\alpha_{n}\right) u_{n}\right)\right\| \\
& =\lim _{n \rightarrow \infty}\left(1-\alpha_{n}\right)\left\|x_{n}-u_{n}\right\| .
\end{aligned}
$$

By the assumption, we have $\lim \sup _{n \rightarrow \infty} \alpha_{n}<1$, we obtain

$$
\left\|x_{n}-u_{n}\right\| \rightarrow 0, \quad \text { as } n \rightarrow \infty
$$

Step 6. We show that $\hat{p} \in \operatorname{GEP}(F, A)$, that is, $J \hat{p} \in J(\operatorname{GEP}(F, A))$. 
From (4.3), (4.5) and (4.6), it follows that

$$
u_{n} \rightarrow \hat{p} \quad \text { and } \quad y_{n} \rightarrow \hat{p}, \quad \text { as } n \rightarrow \infty
$$

From $J$ is uniformly norm-to-norm continuous on bounded subset of $E$, we also have

$$
J u_{n} \rightarrow J \hat{p} \quad \text { and } \quad J y_{n} \rightarrow J \hat{p}, \quad \text { as } n \rightarrow \infty
$$

It follows from (4.5) and (4.6) and the property of $J$ that

$$
\left\|J x_{n}-J y_{n}\right\| \rightarrow 0 \quad \text { and } \quad\left\|J x_{n}-J u_{n}\right\| \rightarrow 0 \quad \text { as } n \rightarrow \infty
$$

Since $\left\{x_{n}\right\}$ is bounded. Therefore, $\left\{u_{n}\right\}$ and $\left\{y_{n}\right\}$ are bounded.

Hence, $\left\{J x_{n}\right\},\left\{J u_{n}\right\}$ and $\left\{J y_{n}\right\}$ are also bounded.

So, there exists a subsequence $\left\{J x_{n_{k}}\right\}$ of $\left\{J x_{n}\right\}$ such that $J x_{n_{k}} \rightarrow \hat{p}^{*}$, and there exists a subsequence $\left\{J u_{n_{k}}\right\}$ of $\left\{J u_{n}\right\}$ such that $J u_{n_{k}} \rightarrow \hat{p}^{*}$.

From (4.6) and $r_{n} \subset[a, \infty)$, we have

$$
\lim _{n \rightarrow \infty} \frac{1}{r_{n}}\left\|x_{n}-u_{n}\right\|=0
$$

Since $u_{n}=T_{r_{n}} x_{n}$, we have

$$
F\left(J u_{n}, J y\right)+\left\langle A J u_{n}, J y-J u_{n}\right\rangle+\frac{1}{r_{n}}\left\langle u_{n}-x_{n}, J\left(y-u_{n}\right)\right\rangle \geq 0, \quad \forall y \in C .
$$

It follows from (DA2) that

$$
\left\langle A J u_{n}, J y-J u_{n}\right\rangle+\frac{1}{r_{n}}\left\langle u_{n}-x_{n}, J\left(y-u_{n}\right)\right\rangle \geq-F\left(J u_{n}, J y\right) \geq F\left(J y, J u_{n}\right), \quad \forall y \in C .
$$

From $x_{n} \rightarrow \hat{p}$ and $u_{n} \rightarrow \hat{p}$, we get

$$
\langle A J \hat{p}, J y-J \hat{p}\rangle+\frac{1}{r_{n}}\langle\hat{p}-\hat{p}, J(y-\hat{p})\rangle \geq F(J y, J \hat{p}), \quad \forall y \in C .
$$

Therefore,

$$
\langle A J \hat{p}, J y-J \hat{p}\rangle \geq F(J y, J \hat{p}), \quad \forall y \in C
$$

For any $0<t<1, y \in C$ and setting $y_{t}^{*}=t J y+(1-t) J \hat{p}$. Then we get $y_{t}^{*} \in J(C)$, and so

$$
\left\langle A J \hat{p}, y_{t}^{*}-J \hat{p}\right\rangle \geq F\left(y_{t}^{*}, J \hat{p}\right), \quad \forall y \in C .
$$

It follows from (DA1) and (DA4) that

$$
\begin{aligned}
0 & =F\left(y_{t}^{*}, y_{t}^{*}\right) \\
& \leq t F\left(y_{t}^{*}, J y\right)+(1-t) F\left(y_{t}^{*}, J \hat{p}\right) \\
& \leq t F\left(y_{t}^{*}, J y\right)+(1-t)\left\langle A J \hat{p}, y_{t}^{*}-J \hat{p}\right\rangle .
\end{aligned}
$$


Letting $t \downarrow 0$, we obtain from assumption (DA3) that

$$
F(J \hat{p}, J y)+\left\langle A J \hat{p}, y_{t}^{*}-J \hat{p}\right\rangle \geq 0 .
$$

This implies that

$$
J \hat{p} \in J(\operatorname{GEP}(F, A))
$$

Step 7. We show that the sequence $\left\{x_{n}\right\}$ converges strongly to $R_{\mathrm{GEP}(F, A)}\left(x_{0}\right)$.

We know that $x_{n}=R_{C_{n}}\left(x_{0}\right) \in \operatorname{GEP}(F, A) \subset C_{n}$.

Let $w=R_{\mathrm{GEP}(F, A)}\left(x_{0}\right)$. It follows from Lemma 2.9 that

$$
\phi\left(x_{0}, x_{n}\right) \leq \phi\left(x_{0}, w\right)
$$

Since the norm is weakly lower semicontinuous, and from (DA4), we have

$$
\begin{aligned}
\phi\left(x_{0}, J^{-1} \hat{p}^{*}\right) & =\left\|x_{0}\right\|^{2}-2\left\langle x_{0}, J\left(J^{-1} \hat{p}^{*}\right)\right\rangle+\|\hat{p}\|^{2} \\
& =\left\|x_{0}\right\|^{2}-2\left\langle x_{0}, \hat{p}^{*}\right\rangle+\|\hat{p}\|^{2} \\
& \leq \liminf _{k \rightarrow \infty}\left(\left\|x_{0}\right\|^{2}-2\left\langle x_{0}, J x_{n_{k}}\right\rangle+\left\|x_{n_{k}}\right\|^{2}\right) \\
& =\liminf _{k \rightarrow \infty} \phi\left(x_{0}, x_{n_{k}}\right) \\
& \leq \limsup _{k \rightarrow \infty} \phi\left(x_{0}, x_{n_{k}}\right) \\
& \leq \phi\left(x_{0}, w\right) .
\end{aligned}
$$

From the definition of $R_{\mathrm{GEP}(F, A)}$, we have $J^{-1} \hat{p}^{*}=w$.

Finally, we show that $x_{n} \rightarrow w$, where $w=R_{\mathrm{GEP}(F, A)}\left(x_{0}\right)$.

Now, we have

$$
\phi\left(x_{0}, x_{n}\right) \leq \phi\left(x_{0}, w\right)=\phi\left(x_{n}, R_{\mathrm{GEP}(F, A)}\left(x_{0}\right)\right),
$$

and from Remark 1.5(2), we get

$$
\phi\left(w, x_{n}\right)=\phi\left(w, x_{0}\right)+\phi\left(x_{0}, x_{n}\right)+2\left\langle w-x_{0}, J x_{0}-J x_{n}\right\rangle .
$$

Therefore,

$$
\begin{aligned}
\limsup _{n \rightarrow \infty} \phi\left(w, x_{n}\right) & =\limsup _{n \rightarrow \infty}\left(\phi\left(w, x_{0}\right)+\phi\left(x_{0}, x_{n}\right)+2\left\langle w-x_{0}, J x_{0}-J x_{n}\right\rangle\right) \\
& \leq \limsup _{n \rightarrow \infty}\left(\phi\left(w, x_{0}\right)+\phi\left(x_{0}, w\right)+2\left\langle w-x_{0}, J x_{0}-J x_{n}\right\rangle\right) \\
& =\phi\left(w, x_{0}\right)+\phi\left(x_{0}, w\right)+2\left\langle w-x_{0}, J x_{0}-J w\right\rangle \\
& =\phi(w, w)=0 .
\end{aligned}
$$

This implies that

$$
\lim _{n \rightarrow \infty} \phi\left(w, x_{n}\right)=0 .
$$


By Lemma 2.10, we get

$$
\lim _{n \rightarrow \infty}\left\|w-x_{n}\right\|=\lim _{n \rightarrow \infty}\left\|R_{\mathrm{GEP}(F, A)}\left(x_{0}\right)-x_{n}\right\|=0 .
$$

Hence,

$$
x_{n} \rightarrow R_{\mathrm{GEP}(F, A)}\left(x_{0}\right), \quad \text { as } n \rightarrow \infty .
$$

Therefore, the sequence $\left\{x_{n}\right\}$ converges strongly to $R_{\mathrm{GEP}(F, A)}\left(x_{0}\right)$. This completes the proof.

If we substitute $x=x_{n}$ and $z=u_{n}$ in equation (4.1), then we obtain the following result which extends the following results by Takahashi and Zembayashi [1] from an equilibrium problem to a generalized equilibrium problem.

Corollary 4.2 Let E be a uniformly smooth, strictly convex and reflexive real Banach space which has a Kadec-Klee property, let $C$ be a nonempty, closed and convex subset of $E$, and let $J$ be the duality mapping from $E$ into $E^{*}$ such that $J(C)$ is closed and convex of $E^{*}$, let us assume that a bifunction $F: J(C) \times J(C) \rightarrow \mathbb{R}$ satisfies the following conditions (DA1)(DA4), let $C^{*}$ be a nonempty, closed and convex subset of $E^{*}$, and let $A: C^{*} \rightarrow E$ be an $\alpha$-inverse strongly skew monotone. Suppose that $\operatorname{GEP}(F, A) \neq \emptyset$. Let $\left\{x_{n}\right\}$ be a sequence generated by

$$
\left\{\begin{array}{l}
x_{0} \in C \quad \text { chosen arbitrary, } \quad C_{0}=C, \\
u_{n} \in C \quad \text { such that } \\
F\left(J u_{n}, J y\right)+\left\langle A J u_{n}, J y-J u_{n}\right\rangle+\frac{1}{r_{n}}\left\langle u_{n}-x_{n}, J\left(y-u_{n}\right)\right\rangle \geq 0, \quad \forall y \in C, \\
y_{n}=\alpha_{n} x_{n}+\left(1-\alpha_{n}\right) u_{n}, \\
C_{n+1}=\left\{z \in C_{n}: \phi\left(y_{n}, z\right) \leq \phi\left(x_{n}, z\right)\right\}, \\
x_{n+1}=R_{C_{n+1}}\left(x_{0}\right), \quad \forall n \geq 0,
\end{array}\right.
$$

where J is the duality mapping on $E,\left\{\alpha_{n}\right\}$ is the sequence in $[0,1]$ such that lim $\sup _{n \rightarrow \infty} \alpha_{n}<$ $1,\left\{r_{n}\right\} \subset[a, \infty)$ for some $a>0$ and $R_{C_{n+1}}$ is the sunny generalized nonexpansive retraction from $E$ onto $C_{n+1}$. Then the sequence $\left\{x_{n}\right\}$ converges strongly to $R_{\mathrm{GEP}(F, A)}\left(x_{0}\right)$, where $R_{\mathrm{GEP}(F, A)}$ is the sunny generalized nonexpansive retraction from $E$ onto $\operatorname{GEP}(F, A)$.

If we set $A \equiv 0$ in Corollary 4.2, then we obtain the following result which extends the following results by Takahashi and Zembayashi [1].

Corollary 4.3 Let E be a uniformly smooth, strictly convex and reflexive real Banach space which has Kadec-Klee property, let $C$ be a nonempty, closed and convex subset of $E$, and let $J$ be the duality mapping from $E$ into $E^{*}$ such that $J(C)$ is closed and convex of $E^{*}$, let us assume that a bifunction $F: J(C) \times J(C) \rightarrow \mathbb{R}$ satisfies the following conditions (DA1)-(DA4). Suppose that $\mathrm{EP}(F) \neq \emptyset$. Let $\left\{x_{n}\right\}$ be a sequence generated 
by

$$
\left\{\begin{array}{l}
x_{0} \in C \quad \text { chosen arbitrary, } \quad C_{0}=C, \\
u_{n} \in C \quad \text { such that } \quad F\left(J u_{n}, J y\right)+\frac{1}{r_{n}}\left\langle u_{n}-x_{n}, J\left(y-u_{n}\right)\right\rangle \geq 0, \quad \forall y \in C, \\
y_{n}=\alpha_{n} x_{n}+\left(1-\alpha_{n}\right) u_{n}, \\
C_{n+1}=\left\{z \in C_{n}: \phi\left(y_{n}, z\right) \leq \phi\left(x_{n}, z\right)\right\}, \\
x_{n+1}=R_{C_{n+1}}\left(x_{0}\right), \quad \forall n \geq 0,
\end{array}\right.
$$

where $J$ is the duality mapping on $E,\left\{\alpha_{n}\right\}$ is the sequence in $[0,1]$ such that $\lim _{\sup } \operatorname{si\infty }_{n \rightarrow \infty} \alpha_{n}<$ $1,\left\{r_{n}\right\} \subset[a, \infty)$ for some $a>0$ and $R_{C_{n+1}}$ is the sunny generalized nonexpansive retraction from $E$ onto $C_{n+1}$. Then the sequence $\left\{x_{n}\right\}$ converges strongly to $R_{\mathrm{EP}(F)}\left(x_{0}\right)$, where $R_{\mathrm{EP}(F)}$ is the sunny generalized nonexpansive retraction from $E$ onto $\mathrm{EP}(F)$.

If we set $F \equiv 0$ in Corollary 4.2 , then Corollary 4.2 is reduced to the following corollary.

Corollary 4.4 Let E be a uniformly smooth, strictly convex and reflexive real Banach space which has a Kadec-Klee property, let $C$ be a nonempty, closed and convex subset of $E$ such that $J(C)$ is closed and convex of $E^{*}$, let $C^{*}$ be a nonempty, closed and convex subset of $E^{*}$ and $A: C^{*} \rightarrow E$ be an $\alpha$-inverse strongly skew monotone. Suppose that $\operatorname{VI}(J(C), A) \neq \emptyset$. Let $\left\{x_{n}\right\}$ be a sequence generated by

$$
\left\{\begin{array}{l}
x_{0} \in C \quad \text { chosen arbitrary, } \quad C_{0}=C, \\
u_{n} \in C \quad \text { such that }\left\langle A J u_{n}, J y-J u_{n}\right\rangle+\frac{1}{r_{n}}\left\langle u_{n}-x_{n}, J\left(y-u_{n}\right)\right\rangle \geq 0, \quad \forall y \in C, \\
y_{n}=\alpha_{n} x_{n}+\left(1-\alpha_{n}\right) u_{n}, \\
C_{n+1}=\left\{z \in C_{n}: \phi\left(y_{n}, z\right) \leq \phi\left(x_{n}, z\right)\right\}, \\
x_{n+1}=R_{C_{n+1}}\left(x_{0}\right), \quad \forall n \geq 0,
\end{array}\right.
$$

where $J$ is the duality mapping on $E,\left\{\alpha_{n}\right\}$ is the sequence in $[0,1]$ such that $\lim _{\sup } \operatorname{su}_{n \rightarrow \infty} \alpha_{n}<$ $1,\left\{r_{n}\right\} \subset[a, \infty)$ for some $a>0$, and $R_{C_{n+1}}$ is the sunny generalized nonexpansive retraction from $E$ onto $C_{n+1}$. Then the sequence $\left\{x_{n}\right\}$ converges strongly to $R_{\mathrm{VI} U(C), A)}\left(x_{0}\right)$, where $R_{\mathrm{VI}(J(C), A)}$ is the sunny generalized nonexpansive retraction from $E$ onto $\mathrm{VI}(J(C), A)$.

\section{Competing interests}

The authors declare that they have no competing interests.

\section{Authors' contributions}

All authors contributed equally and significantly in writing this article. All authors read and approved the final manuscript.

\section{Author details}

'Department of Mathematics, Faculty of Science, King Mongkut's University of Technology Thonburi (KMUTT), Bang Mod, Thung Khru, Bangkok, 10140, Thailand. ²Department of Mathematics, Faculty of Education, Bansomdejchaopraya Rajabhat University (BSRU), Thonburi, Bangkok, 10600, Thailand.

\section{Acknowledgements}

The first author would like to thank the Bansomdejchaopraya Rajabhat University for financial support. The authors would like to thank the Higher Education Research Promotion and National Research University Project of Thailand's Office of the Higher Education Commission for financial support (Under NRU-CSEC Project No. NRU56000508). 


\section{References}

1. Takahashi, W, Zembayashi, K: A strong convergence theorem for the equilibrium problem with a bifunction defined on the dual space of a Banach space. In: Proceedings of the 8th International Conference on Fixed Point Theory and Its Applications, pp. 197-209. Yokohama Publisher, Yokohama (2007)

2. Blum, E, Oettli, W: From optimization and variational inequalities to equilibrium problems. Math. Stud. 63, 123-145 (1994)

3. Aoyama, K, Kimura, Y, Takahashi, W: Maximal monotone operators and maximal monotone functions for equilibrium problems. J. Convex Anal. 15, 395-409 (2008)

4. Cioranescu, I: Geometry of Banach spaces, duality mappings and nonlinear problem. In: Hazewinkel, M (ed.) Mathematics and Its Applications, vol. 62. Kluwer Academic, Dordrecht (1990)

5. Takahashi, W: Nonlinear Functional Analysis. Fixed Point Theory and Its Application. Yokohama Publisher, Yokohama (2000)

6. Alber, YI: Metric and generalized projection operators in Banach space: properties and applications. In: Kartosator, AG (ed.) Theory and Applications of Nonlinear Operators of Accretive and Monotone Type. Lecture Notes in Pure and Applied Mathematics, vol. 178, pp. 15-50. Marcel Dekker, New York (1996)

7. Kamimura, S, Takahashi, W: Strong convergence of a proximal-type algorithm in a Banach space. SIAM J. Optim. 13(3), 938-945 (2002)

8. Plubtieng, S, Sriprad, W: Existence and approximation of solution of the variational inequality problem with a skew monotone operator defined on the dual spaces of Banach spaces. J. Nonlinear Anal. Optim. 1, 23-33 (2010)

9. Ibaraki, T, Takahashi, W: A new projection and convergence theorems for the projections in Banach spaces. J. Approx. Theory 149, 1-14 (2007)

10. liduka, $\mathrm{H}$, Takahashi, W: Weak convergence theorems for a projection algorithm for variational inequalities in Banach spaces. J. Math. Anal. Appl. 339, 668-679 (2008)

11. Honda, T, Takahashi, W, Yao, JC: Nonexpansive retraction onto closed convex cones in Banach spaces. Taiwan. J. Math 14, 1023-1046 (2010)

12. Kohsaka, F, Takahashi, W: Generalized nonexpansive retractions and a proximal-type algorithm in Banach spaces. J. Nonlinear Convex Anal. 8, 197-209 (2007)

13. Chang, S, Lee, HWJ, Chan, CK, Yang, L: Approximation theorems for total quasi- $\boldsymbol{\phi}$-asymptotically nonexpansive mapping with applications. Appl. Math. Comput. 218, 2921-2931 (2011)

14. Tan, KK, Xu, HK: Approximating fixed points of nonexpansive mappings by the Ishikawa iteration process. J. Math. Anal. Appl. 178, 301-308 (1993)

15. Kanster, B, Kuratowski, C, Mazurkiewic, S: Ein beweis des fixpunktsatzes für $n$-dimensionale simplexe. Fundam. Math $14,132-137$ (1929)

16. Fan, K: A generalization of Tychonoffs fixed point theorem. Math. Ann. 142, 305-310 (1961)

10.1186/1687-1812-2013-264

Cite this article as: Phuangphoo and Kumam: Existence and approximation for a solution of a generalized equilibrium problem on the dual space of a Banach space. Fixed Point Theory and Applications 2013, 2013:264

\section{Submit your manuscript to a SpringerOpen ${ }^{\circ}$ journal and benefit from:}

- Convenient online submission

- Rigorous peer review

- Immediate publication on acceptance

- Open access: articles freely available online

- High visibility within the field

- Retaining the copyright to your article 